http://revistas.ucr.ac.cr/index.php/ingenieria www.ucr.ac.cr / ISSN 2215-2652

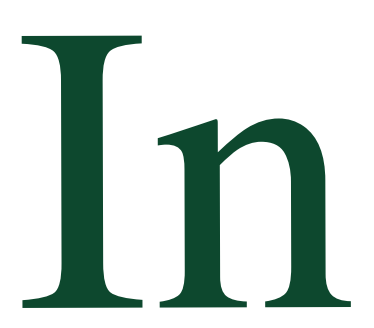

○

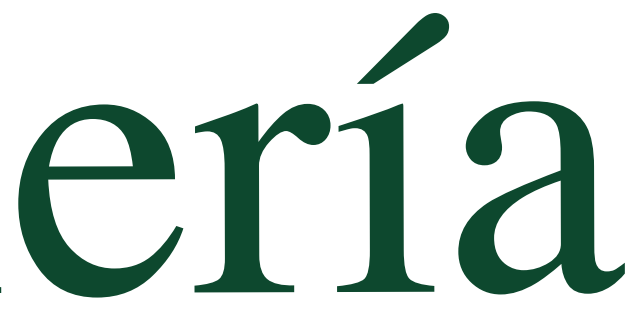

Revista de la Universidad de Costa Rica ENERO / JUNIO 2021 - VOLUMEN 31 (1) 


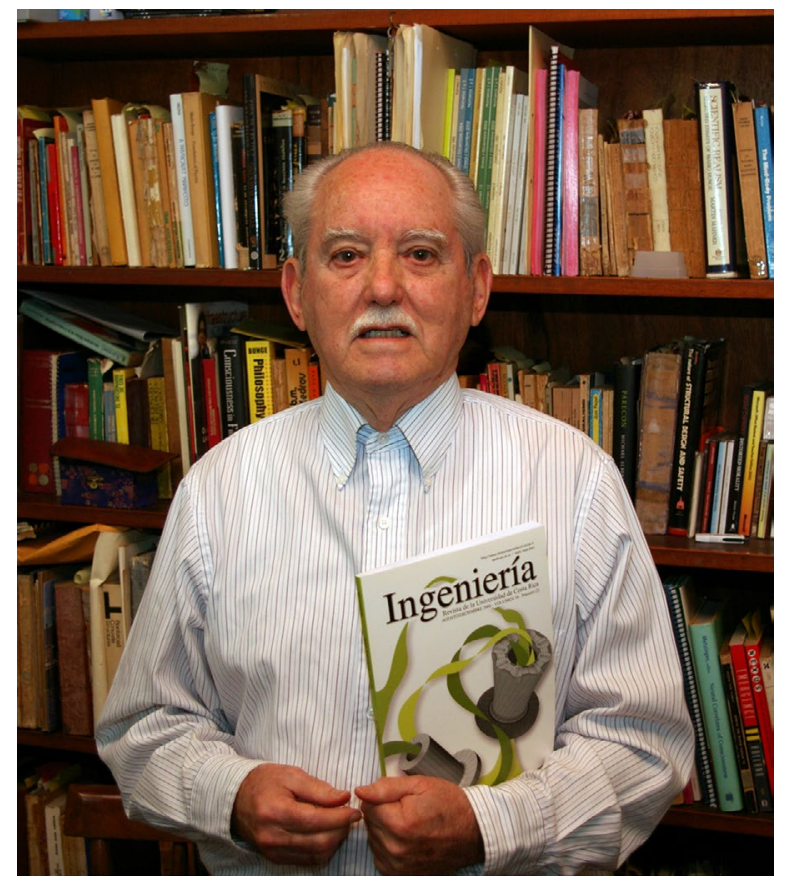

Ing. Rodolfo Herrera en la biblioteca de su casa de habitación. Fuente: fotografía de Alex Fernández, funcionario del CEDAA, INII, UCR.

\section{Dr. Rodolfo Herrera Jiménez, maestro de ingenieros: Una breve semblanza}

\author{
Ph.D. Rodolfo Herrera \\ Jiménez, Master of Engineers:
}

A Brief History

Diego A. Hidalgo Leiva

Director, Revista Ingeniería

e-mail:diego.hidalgo@ucr.ac.cr

ORCID: 0000-0003-3662-4969

\title{
Resumen
}

El 17 de noviembre del 2018 falleció el Dr. Rodolfo Herrera Jiménez, quién será recordado por su calidad humana, su visión y sus aportes tanto a la academia universitaria como a la práctica de la ingeniería estructural y sísmica de Costa Rica. El pasado 10 de julio del 2019, la Comisión Permanente del Código Sísmico de Costa Rica realizó un homenaje póstumo al Doctor Herrera, donde participaron miembros y exmiembros del Código Sísmico de Costa Rica, compañeros de la Universidad de Costa Rica, su esposa y sus hijos. El comité editorial de esta revista ha considerado importante documentar parte de las intervenciones realizadas ese día en combinación con remembranzas de personas allegadas al Dr. Herrera.

\begin{abstract}
On November 17, 2018, PhD Rodolfo Herrera Jiménez passed away. He will be remembered for his quality as a person, his vision, and his contributions both to the university and to the practice of structural and seismic engineering in Costa Rica. On July 10, 2019, the Permanent Commission of the Seismic Code of Costa Rica made a posthumous tribute to PhD Herrera, where members and former members of the Costa Rican Seismic Code, colleagues from the University of Costa Rica, his wife and sons participated. The editorial committee of this Journal has considered important to document part of the interventions carried out that day in combination with remembrances of people close to $\mathrm{PhD}$. Herrera.
\end{abstract}


La revista Ingeniería de la Universidad de Costa Rica, en su celebración por los 30 años de actividad en el proceso de divulgación científica, ha querido realizar un homenaje póstumo a quien fuera miembro fundador y honorario de la revista. Trabajó de manera incansable por lograr una difusión de calidad desde la dirección de la misma entre los años 1989 y el 2001; asimismo, publicó el primer número de la revista en 1991. He aceptado la difícil tarea de hacer justicia a la memoria de este gran maestro cuyos aportes cimentaron la estructura de la actual Facultad de Ingeniería, así como la Comisión Permanente de Estudio del Código Sísmico de Costa Rica; instituciones cuyo aporte a la sociedad costarricense es innegable.

El Ing. Herrera estuvo entre las aulas de la Universidad de Costa Rica desde 1950 cuando ingresó a estudiar Ingeniería Civil. Como nos ha pasado a muchos, la Universidad de Costa Rica se convirtió para don Rodolfo en una segunda casa. Poco después de su graduación se reincorpora a la academia, primero en la Escuela de Física-Matemática para posteriormente integrarse al cuerpo docente de la Escuela de Ingeniería Civil desde 1962 hasta el momento en que se acoge a su pensión. Don Rodolfo disfrutaba los cursos del área de la mecánica racional, por lo que se le recuerda por impartir cursos como Mecánica Teórica, Mecánica de Materiales y Mecánica de Estructuras, con una claridad admirable.

Por su parte, respecto al área administrativa de la Universidad, fue director en dos periodos distintos de la Escuela de Ingeniería Civil (1965-1968 y 1972-1978), donde ayudó a modernizar el plan de estudios para pasar de 6 años a 5 años y además se pasó de cursos anuales a semestrales, temas hoy superados y considerados como parte de la definición de la carrera. Entre 1982 y 1990 fue Decano de la Facultad de Ingeniería y además fue director de la Cátedra Luis González, que se inauguró en 1986. Finalmente, fue miembro del Consejo Director del Posgrado en Ciencias Cognitivas. Después de pensionarse, siguió vinculado a la Universidad como Profesor Emérito de la Escuela de Ingeniería Civil.

Con 12 publicaciones científicas en la Revista Ingeniería, el Dr. Herrera es uno de los autores con más contribuciones a lo largo de los 30 años de la revista. Es necesario resaltar la variedad de temas que, dada la formación tan diversa que poseía, era capaz de abarcar con facilidad y solvencia. Los principales temas de investigación del Dr. Herrera se centraban en la mecánica de estructuras [1] y la ingeniería sísmica en Costa Rica [2]. Asimismo, aportó tanto en el área filosófica de la ingeniería[3], como en la formación académica y sus sistemas educativos [4], para finalmente llegar a discutir temas de áreas afines de otras ingenierías como la Ingeniería Eléctrica [5].

Don Rodolfo Herrera ayudó a formar muchas generaciones de ingenieros, las cuales han contribuido al desarrollo del país, pero además fue pieza clave en la formulación de la primera versión del Código Sísmico de Costa Rica [6], así como de la creación de la primera Comisión Permanente de Estudio del Código Sísmico. Don Rodolfo fue un profesor incansable, que pasó de enseñar en las aulas a compartir sus conocimientos ya no solo con sus estudiantes, sino con colegas y amigos, quienes nunca dejaron de ver en él a un maestro de la Ingeniería.

Hoy más que nunca necesitamos personas como don Rodolfo. Debemos de ver más allá de la disciplinariedad en nuestras profesiones y dar el paso a la trans/multi/pluri-disciplinariedad, 
pues los grandes problemas que debemos resolver como humanidad serán mejor entendidos en la combinación de conocimientos, en la atención a los detalles y en la apertura a compartir aquello que sabemos sin esperar nada más que el beneficio de la sociedad.

Desde la Revista Ingeniería queremos dar las gracias a don Rodolfo por el legado que nos ha dejado. Nos comprometemos a buscar siempre la excelencia y poner en alto el estandarte que nos ha pasado. Siempre será recordado.

Agradezco a la Ingeniera Marcia Cordero Sandí por concederme el discurso dado en el acto de la Comisión Permanente del Código Sísmico, en representación de la Escuela de Ingeniería Civil de la Universidad de Costa Rica, utilizado como referencia para esta semblanza.

\section{REFERENCIAS}

[1] R. Herrera Jiménez, “Analisis espacial de edificios”, Revista Ingeniería, vol. 7, no. 2, pp. 9-39, 1998, doi: $10.15517 /$ ring.v7i2.7694.

[2] R. Herrera Jiménez, “Amenaza sísmica y el Código Sísmico de Costa Rica,” Revista Ingeniería., vol. 10, no. 1, pp. 107-124, 2000.

[3] R. Herrera Jiménez, "Sistema y lo sistémico en el pensamiento contemporaneo," Revista Ingeniería., vol. 17, no. 11-26, 2007.

[4] R. Herrera Jiménez, "Universidad, Sistemas educativos y carreras acadámicas: Un enfoque sistémico," Revista Ingeniería., vol. 17, no. 2, pp. 13-36, 2008.

[5] R. Herrera Jiménez, "Las Leyes De Kirchhoff Y Las Redes Eléctricas De Corriente Continua," Revista Ingeniería, vol. 13, no. 1-2, 2011, doi: 10.15517/ring.v13i1-2.622.

[6] CFIA, Código Sísmico de Costa Rica 1974, 1ra ed. Colegio Federado de Ingenieros y Arquitectos CFIA, 1974. 